\title{
The influence of time interval between diagnostic image acquisition and operative date on pathologic tumor size in pancreatic adenocarcinoma: implications for local therapy
}

\author{
Caleb R. Dulaney ${ }^{1}$, William A. Hall' ${ }^{1}$, J ohn L. Mikell ${ }^{1}$, Pardeep Mittal ${ }^{2}$, Roshan S. Prabhu ${ }^{1}$, David A. \\ Kooby $^{3}$, Krisztina Hanley ${ }^{4}$, Juan M. Sarmiento ${ }^{5}$, Jerome C. Landry ${ }^{1}$ \\ 1. Departments of Radiation Oncology and Winship Cancer Institute, Emory University, Atlanta, Georgia, USA. 2. \\ Departments of Radiology and Winship Cancer Institute, Emory University, Atlanta, Georgia, USA. 3. Departments of \\ Surgical Oncology and Winship Cancer Institute, Emory University, Atlanta, Georgia, USA. 4. Departments of Pathology \\ and Winship Cancer Institute, Emory University, Atlanta, Georgia, USA. 5. Departments of General Surgery and Winship \\ Cancer Institute, Emory University, Atlanta, Georgia, USA.
}

Correspondence: Jerome C. Landry. Address: Departments of Radiation Oncology and Winship Cancer Institute, 1365 Clifton Road NE, Atlanta, USA. E-mail: Jland01@emory.edu

Received: July 17, 2013

DOI : $10.5430 / j b g c . v 4 n 2 p 15$
Accepted: August 18, 2013

Online Published: February 21, 2014

\section{Abstract}

Objectives: Computed tomography (CT) and magnetic resonance imaging (MRI) may underestimate pancreatic tumor size, which has important implications for local therapy. Our aim was to determine if tumor growth during the interval between image acquisition and operative date impacted the observed size discrepancy.

Methods: Tumor sizes measured on preoperative MRI were compared with gross pathological specimen measurements in 148 patients with surgically resected pancreatic adenocarcinoma. Differences in the measurements were correlated with the interval between date of pre-operative MRI acquisition and date of operation. Differences between tumor size on MRI and pathology reports were compared with respect to the intervening time interval.

Results: A total of 148 patients had pre-operative MRI scans and were included in the analysis. The median patient age was 66 years (range: 29 years-86 years). A significant under estimation of $4.5 \mathrm{~mm}$ between tumor size measured on preoperative MRI and pathological examination $(p<.001)$ was demonstrated. There was no significant correlation between size discrepancy and time interval from the diagnostic imaging study and the surgical procedure $\left(\mathrm{R}^{2}=0.001\right.$, $p=.72)$.

Conclusions: Time interval between the acquired diagnostic imaging study and operative date appears to have no measureable influence on radiographic to pathologic size discrepancy in pancreatic adenocarcinoma. MRI was again shown to underestimate pancreatic cancer tumor size. Additional exploration into the role of MRI in delineating pancreatic tumor volume with a prospectively designed study is needed to validate these findings.

\section{Key words}

Pancreatic adenocarcinoma, Pancreatic cancer and radiation therapy, Magnetic resonance imaging for pancreatic cancer, Radiotherapy planning for pancreatic cancer 


\section{Introduction}

Pancreatic Adenocarcinoma (PAC) remains a devastating malignancy with overall 5-year survival rates of less than $5 \%{ }^{[1]}$. These tumors are seated deep in the retroperitoneum and frequently involve neurovascular structures critical to the function of the gastrointestinal (GI) system. Consequently, PAC often presents at an advanced stage. Nearly $50 \%$ of patients present with distant metastasis, $30 \%$ have unresectable disease, and only $20 \%$ have a tumor amenable to surgery. Of those patients able to undergo surgery, the 5-year overall survival (OS) ranges from 15\%-27\%, and local recurrence remains a relatively common problem ${ }^{[2-4]}$. For those patients with unresectable disease, a combination of radiotherapy (RT) and chemotherapy offers the best option for local control and possible prolonged survival ${ }^{[5-6]}$.

Recent early phase clinical trials have applied high dose stereotactic body radiation therapy (SBRT) treatments for patients with locally advanced PAC, demonstrating impressive rates of local control ${ }^{[7-9]}$. There is emerging data on genetic markers that help determine which patients are more likely to develop distant metastatic disease ${ }^{[10]}$. As these genetic markers are further validated and systemic therapies improve, local control in PAC will play an even greater role in overall disease control and survival.

Essential to the process of conformal RT in any disease site is the accurate delineation of gross tumor volume (GTV). In PAC it is currently unknown which imaging modality is best for tumor volume delineation. Recent data has emerged showing that computed tomography (CT) underestimates tumor size by a median of $7 \mathrm{~mm}^{[11]}$. Advanced MRI sequences have shown only minimal advantages for tumor size delineation over $\mathrm{CT}^{[14]}$. Given this consistent radiographic to pathologic size discrepancy, determining factors that contribute to this discrepancy is important for accurate RT delivery. Several series speculate that tumor growth in the interval between the original diagnostic imaging study and surgical resection could contribute to the underestimation of tumor size on CT compared with surgical pathology ${ }^{[11-13]}$. This poses the question: in the setting of unresectable disease if a prolonged time interval has elapsed between the initial diagnostic scan and surgical resection, does that initial scan merit repeating for the purposes of GTV delineation?

The primary objective of this study was to determine the influence of time interval between imaging and operation on the observed radiographic to pathologic discrepancy of tumor size as measured on magnetic resonance imaging (MRI) and gross pathologic specimen ${ }^{[14]}$.

\section{Methods and materials}

\subsection{Patient selection}

The records of 209 consecutive patients with resected PAC and preoperative MRI scans performed between 2006 and 2011 at Emory University were reviewed. A total of 148 patients were found to meet the following inclusion criteria: preoperative MRI with a report of largest tumor dimension, complete pathologic report documenting PAC with at least one tumor dimension, and no therapy prior to resection. Dimensions from radiology reports documenting tumor size were recorded along with the largest dimension in the pathology report. As these dimensions were taken from clinical reports, multiple observers were involved in the measurement process. This review was approved by the Emory University Institutional Review Board prior to any data collection or analysis.

\subsection{MRI acquisition}

MRI examinations were performed with a 1.5-T MRI scanner (Magnetom Avanto [Siemens Medical Solutions, Erlangen, Germany]; Gyroscan Intera [Philips Medical Systems, Best, the Netherlands]; or Twin-EXCITE [GE Healthcare, Milwaukee, WI]). A torso phased-array surface coil was used for signal reception. Routine for each set of MRI scans included multisequence unenhanced in the axial plane and selected coronal imaging. The axial sequences were as follows: T1-weighted breath-hold spoiled GRE dual echo in- and opposed-phase (repetition time [TR]/echo time [TE], 
120Y170/2.2Y4.4; flip angle, 80Y90 degrees); T2-weighted partial Fourier single-shot echo train (effective TR, infinite; effective TE, 80 Y 90 milliseconds). The section thickness was $2 \mathrm{~mm}$ to $3 \mathrm{~mm}$, and the matrix size was 128 to $192 \times 256$ (phase $\times$ frequency) for all sequences. A power injector (Medrad, Indianola, $\mathrm{Pa}$ ) was used to administer intravenous $\mathrm{Gd}$ to all patients with a bolus of $0.05 \mathrm{mmol} / \mathrm{kg}$ of Gd (MultiHance, Bracco, Milan, Italy) at $2 \mathrm{ml} / \mathrm{s}$ followed by a 20 -ml saline flush at the $2 \mathrm{ml} / \mathrm{s}$. A total of four sets of breath hold serial axial fat-saturated T1-weighted 3D GRE images were acquired before contrast administration and during the contrast-enhanced tissue arterial dominant phase, venous phase, and interstitial phases. Acquisition parameters were TR/TE, 3.4Y3.8/1.2Y1.4; flip angle, 12 degrees; acceleration factor, 2; section thickness, $2 \mathrm{~mm}$ to $3 \mathrm{~mm}$; matrix size, $192 \times 256$; field of view, $400 \mathrm{~mm}$ (rectangular field of view, 75\%); scan percentage, $80 \%$; partial Fourier imaging in the phase and slice directions. The arterial phase images were acquired with a real-time-bolus-tracking method triggered eight seconds after the arrival of contrast medium in the celiac arterial axis, during which time the patient was given breath-hold instructions. The venous and interstitial phase images were acquired 70 seconds and 180 seconds, respectively, after contrast administration. Acquisition time ranged from 12 seconds to 15 seconds because of the varying fields of view and because the number of sections was individually modified according to patient stature.

\subsection{Statistical analysis}

Differences between tumor size on MRI and pathology were correlated with the time interval between the date of the imaging study and the operation. Statistical analysis was conducted using Microsoft Excel version 2008 (Microsoft Inc, Redmond, WA) and SPSS version 19.0 statistical software package (IBM Inc., Armonk, NY). Tumor size difference was calculated as the maximum size reported by pathological examination minus the maximum size reported by MRI. Previous studies have demonstrated a significant difference between mean tumor sizes measured on CT or MRI and pathological examination ${ }^{[11,13,14]}$. Therefore, mean tumor sizes measured by MRI were compared to those measured by pathological exam to confirm observed discrepancies in our previous report ${ }^{[14]}$. The association of time interval from MRI to operation date with size difference was measured using the Pearson correlation coefficient. The association between time interval and both pathological and MRI size was also measured using the Pearson correlation coefficient. Tumor size differences between MRI and pathology were compared according to time interval. Tumor size differences and time intervals were compared between groups using the Mann Whitney $U$-test. We used an exponential growth equation (Equation 1) to predict tumor growth over the interval from MRI to operation using previously reported median tumor volume doubling times (TVDT) ranging from 46.15 days to 144 days ${ }^{[12,15,16]}$.

\subsection{Measurements}

In Equation 1, $\Delta \mathrm{t}$ represents difference in days between date of imaging and date of operation, D1 represents the predicted maximal tumor diameter, and D0 represents the initial tumor diameter. The predicted tumor sizes were compared to MRI and pathological sizes using a paired $t$-test ${ }^{[12,15,16]}$.

$$
\mathrm{TVDT}=\Delta \mathrm{t} \times \log (2) \div[3 \times \log (\mathrm{D} 1 / \mathrm{D} 0)]
$$

\section{Results}

\subsection{Patient and tumor characteristics}

Patient characteristics are shown in Table 1. Of the 148 included patients, the majority had tumors located in the pancreatic head with a median size on MRI of $2.7 \mathrm{~cm}$ and a median pathologic size of $3.2 \mathrm{~cm}$. All patients underwent surgical resection, with a total of $27 \%$ of patients having positive margins on pathological examination. Median follow-up time was 20.5 months and $30 \%$ of patients had local or distant recurrence in the follow-up period. A total of 16 patients had local failure, 20 had distant failure, and 1 had both local and distant failure. There was a significant difference between tumor size measured by MRI and pathological examination $(p<.001)$. In total, MRI underestimated $67 \%$ of tumor sizes, 
with a median size discrepancy of $4.5 \mathrm{~mm}$. Mean and median time interval between the date of diagnostic imaging and surgical resection were 12.96 days and 8.5 days respectively with a range of one day to 52 days. Figure 1 demonstrates the distribution of time interval between MRI and operation for the patient population.

Table 1. All patients baseline characteristics

\begin{tabular}{llll}
\hline Demographic & N = 148 & & \\
\hline Age & & & \\
Mean & 65.25 & & \\
Median (Range) & $66(29-86)$ & & \\
Gender & & & \\
Male no. (\%) & $65(44)$ & & \\
Operation Minus MRI Date & & & 3.45 \\
Mean & 12.96 & Pathology Tumor Size (cm) & $3.2(1-10)$ \\
Median (Range) & $8.5(1-52)$ & Mean & \\
Tumor Characteristics & & Median (Range) & \\
MRI Tumor Size (cm) & 2.85 & & $93(66.4)$ \\
Mean & $2.7(0.5-9.5)$ & & $47(33.6)$ \\
Median (Range) & & LN Positive \\
Difference (Path - MRI) (cm) & 0.59 & Yes & 8 \\
Mean & $0.45(-2.6-3.8)$ & No & \\
Median (Range) & & Missing & \\
Histologic Grade & $11(7.4)$ & & $122(83)$ \\
Unspecified & $10(6.7)$ & Location \\
I & $86(58.1)$ & Head/Neck & $5(3.4)$ \\
II & $41(27.7)$ & Body & $20(13.6)$ \\
III/IV & & Tail & \\
Margin & $102(72.9)$ & & \\
Negative & $88(27.1)$ & & \\
Positive & & & \\
Missing & & & \\
\hline
\end{tabular}

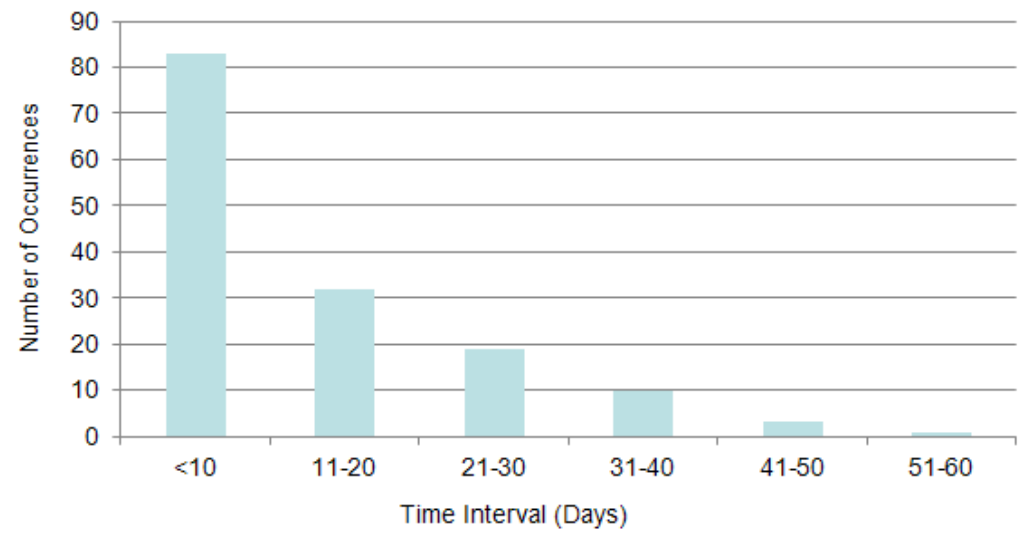

Figure 1. Distribution of intervals from MRI to operation

\subsection{Tumor measurement correlated with time interval}

There was no significant correlation between the size discrepancy on MRI or pathological specimen and the time interval between MRI acquisition date and operative date (see Figure $2\left[\mathrm{R}^{2}=0.001, p=.72\right]$ ). There was also no significant correlation between the tumor size on the MRI and time interval (see Figure $3\left[\mathrm{R}^{2}=0.005, p=.42\right]$ ) or tumor size on pathology and time interval $\left(\mathrm{R}^{2}=0.001, p=.72\right)$. When tumor size discrepancy was compared between those with time interval less than $(0.51 \pm 0.98 \mathrm{~cm})$ and greater than $(0.67 \pm 1.16 \mathrm{~cm})$ the median $(8.5$ days $)$, there was also no significant 
difference measured $(p=.32)$. A subset of 14 patients with imaging performed within one day of operation were compared to a second group of 14 patients with imaging greater than 30 days prior to operation and showed no significant difference in tumor size discrepancy $(0.24 \pm 1.34 \mathrm{~cm}, \leq 1$ day; $0.36 \pm 1.16 \mathrm{~cm}, \geq 30$ days; $p=.91)$. The average time interval for patients with tumors that were underestimated (12.4 \pm 10.9 days) on the diagnostic MRI were compared to those that were overestimated (14.76 \pm 12.06 days) and showed no difference in time interval between imaging and surgery based on direction of tumor size discrepancy $(p=.26)$.

Figure 2. Plot of time interval between imaging and operation versus size difference between pathology and MRI showing no significant correlation

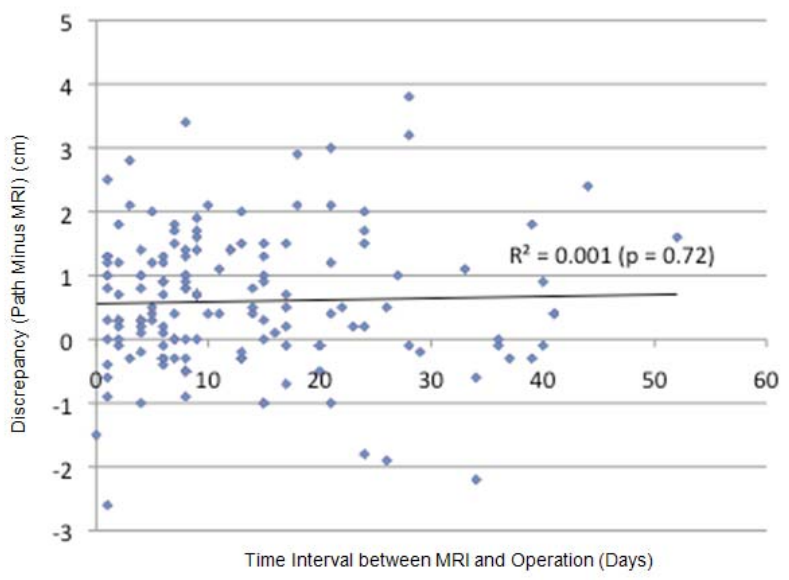

Figure 3. Plot of time interval from imaging to operation versus tumor size measured on (A) MRI, and (B) Pathological Examination

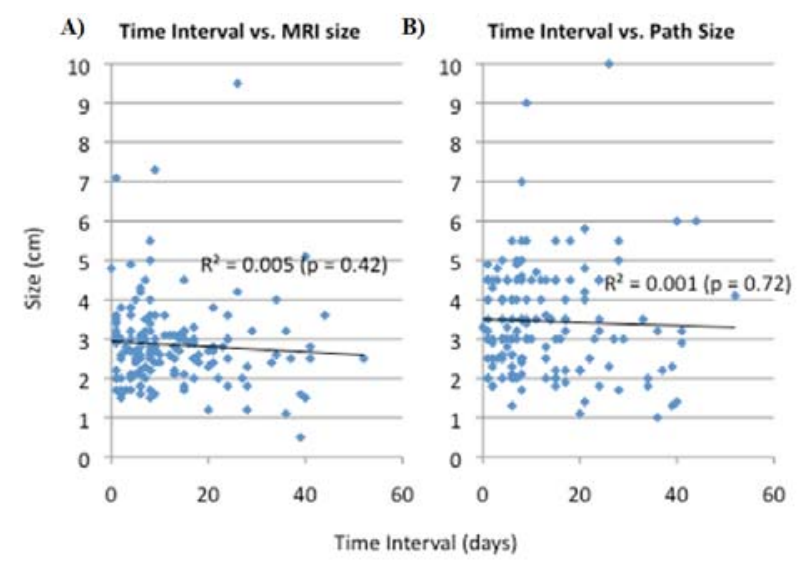

\subsection{Calculation of TVDT}

Table 2 shows results from modeling of tumor growth using previously reported TVDTs. When we modeled tumor growth over the time interval between MRI and operation using Equation 1, pathological sizes continued to be significantly larger $(p<.01)$ than the predicted size using all previously reported pancreatic TVDTs, including the shortest reported doubling time of 46.15 days. By contrast, comparison of predicted tumor sizes and sizes measured on MRI demonstrated no significant difference using all TVDTs. Next, we wanted to determine what TVDT would be necessary for pathological tumor sizes to no longer be significantly larger than predicted sizes using our model. Our model predicted a value for TVDT of 31.2 days necessary to eliminate the significant difference between predicted size and pathological size $(p>.05)$.

Table 2. Model of tumor growth over interval from imaging to operation

\begin{tabular}{llllll}
\hline TVDT (days) & Predicted Size $\mathbf{( c m )}$ & MRI Size $\mathbf{( c m )}$ & $\begin{array}{l}\text { Pathological Size } \\
\mathbf{( c m )}\end{array}$ & $\begin{array}{l}\boldsymbol{p} \text {-value } \\
\text { (Predicted vs. MRI) }\end{array}$ & $\begin{array}{l}\boldsymbol{p}^{*} \text {-value } \\
\text { (Predicted vs. Path) }\end{array}$ \\
\hline 46.15 & $3.04 \pm 1.22$ & $2.85 \pm 1.12$ & $3.44 \pm 1.38$ & .16 & .008 \\
86 & $2.95 \pm 1.17$ & - & - & .45 & $\leq .001$ \\
144 & $2.91 \pm 1.15$ & - & - & .66 & $\leq .001$ \\
\hline
\end{tabular}

Note. TVDT $=$ Tumor Volume Doubling Time. ${ }^{*}$ Significance based on $p \leq .05$ using paired $t$-test.

Published by Sciedu Press 


\section{Discussion}

The process of accurate GTV delineation in locally advanced PAC is essential to RT delivery and contributes to improved local control, reduced morbidity, and lower normal tissue toxicity. While there is some evidence showing an acceptable correlation between pancreatic tumor sizes measured by imaging and pathological examination ${ }^{[12]}$ there is also mounting evidence that both CT and MRI underestimate tumor size ${ }^{[1,13,17]}$. In a recent series by Arvold, et al., $84 \%$ of pancreatic tumors were underestimated by $\mathrm{CT}$ with a median size discrepancy of $7 \mathrm{~mm}$ and a range of $-15 \mathrm{~mm}$ to $43 \mathrm{~mm}{ }^{[11]}$. This underestimation by CT was confirmed by Qiu, et al. who compared conventional CT and 3D-CT showing a median underestimation of $3.2 \mathrm{~mm}$ and a large range in the radiographic to pathologic discrepancy ${ }^{[13]}$. The underestimation of tumor size is not unique to CT as Hall et al. have recently presented an analysis showing that MRI also tends to underestimate tumor size when compared with gross pathologic specimen. Many studies examining the radiographic to pathologic size discrepancy raise the question regarding the influence of time between the diagnostic scan and surgery and the role of interval tumor growth in contributing to this discrepancy. The purpose of this study was to examine this question with a large cohort of resected PAC patients with pre-operative MRI's with wide variability in the time between MRI acquisition and surgical resection date.

The importance of tumor growth is paramount to the radiation oncologist in the process of GTV delineation. Our study confirms a previously presented discrepancy between tumor sizes measured on MRI and pathological examination of 4.5 $\mathrm{mm}^{[14]}$. Our results further demonstrate that there is no significant influence of time interval between the acquired imaging study and operation date on the observed radiographic to pathologic discrepancy. Overall, tumor growth does not appear to have any influence on this discrepancy over the range of time intervals presented in our study ( 1 day-52 days). On subgroup analysis we compared patients with imaging acquired one day prior to surgery to those with imaging over 30 days prior to surgery and found no difference in size discrepancy. If tumor growth had any measureable impact on the discrepancy between radiographic and pathologic measurements, we would have expected it to manifest in this subgroup analysis. Furthermore, even the shortest TVDT did not introduce a significant difference between MRI size and predicted size. Therefore, over the course of one month, it appears that tumor growth is not significant enough to introduce the 4-5 $\mathrm{mm}$ size discrepancies seen in this study and prior reports ${ }^{[14]}$.

We have confirmed in this larger patient cohort that there does appear to be a significant size discrepancy between tumor sizes reported on MRI and those reported on pathological examination, which has been previously published by our group in a smaller cohort of patients ${ }^{[14]}$. Our data suggests that pancreatic tumors do not grow significantly over the course of up to one month and this finding may have important implications for the Radiation Oncologists. When applying diagnostic imaging studies that have been acquired over the course of the past month (which is typical in the initial process of pancreatic tumor evaluation) our data show that those studies can be incorporated into the treatment planning process without any substantial adjustment for tumor growth. Furthermore, it is not necessary for those studies to be repeated in close proximity to the treatment date in order to account for additional tumor growth.

This study does have limitations beyond its retrospective design, which should be recognized. In previous analysis, each MRI was individually re-measured by a diagnostic radiologist to help minimize inter-observer bias ${ }^{[14]}$. This step was not repeated in the current study which may have introduced a component of interobserver variability. The measurements from MRI reports were compared to the re-measurements and there was no statistically significant difference shown. Similarly, the pathology measurements were obtained from pathology reports and were not independently reviewed. It is possible that changes in tumor shape and size during specimen processing and examination as well as interobserver variability could have potentially introduced discrepancy between imaging and pathology. Pancreatic tumors also pose a challenge to adequately visualize on radiographic images. Anecdotally, we observed a number of patients in the present study who had either no report of tumor prior to surgery or tumor so poorly defined that size could not be determined. The consistency of radiographic measurements underestimating tumor size among a variety of studies and imaging modalities suggests that our current methods of delineating pancreatic GTV may have inherent inaccuracies. 
Further research is necessary to determine what clinical, radiographic, or histopathological factors cause the observed tumor size discrepancies between imaging and pathological examination. Improvements in imaging protocols to optimize visualization of pancreatic tumors could potentially help eliminate the observed discrepancy. Applying these improvements in imaging modalities could help to more effectively target local RT. It has yet to be determined what effect this discrepancy may have on the ability to accurately cover pancreatic tumor volumes with highly conformal RT treatments or how they should be accounted for in the treatment planning process.

\section{Conclusion}

The optimal imaging modality for delineating pancreatic tumor volume has yet to be determined. Both MRI and CT have been shown to underestimate tumor size when compared with pathological examination. This series has demonstrated that tumor growth in the interval of time between imaging and operation appears to have no measureable influence on this underestimation. Pancreatic tumors do not appear to grow rapidly over the course of two to four weeks. Finally, this study underscores the challenge of adequately visualizing tumors of the pancreas with current imaging techniques. Mechanisms for understanding the true extent of pancreatic GTV require further investigation. As in many other disease sites treated by Radiation Oncologists, multiple imaging modalities should be considered when planning treatment for pancreatic tumors. Further research is needed to determine what clinical, radiographic, and histopathological factors cause current imaging modalities to underestimate pancreatic tumor size.

\section{References}

[1] Jemal A, Siegel R, Xu J, Ward E. Cancer statistics, 2010. CA: a cancer journal for clinicians. 2010; 60: 277-300. PMid: 20610543. http://dx.doi.org/10.3322/caac.20073

[2] Hattangadi JA, Hong TS, Yeap BY, Mamon HJ. Results and patterns of failure in patients treated with adjuvant combined chemoradiation therapy for resected pancreatic adenocarcinoma. Cancer. 2009; 115: 3640-3650. PMid: 19514088. http://dx.doi.org/10.1002/cncr.24410

[3] Griffin JF, Smalley SR, Jewell W, et al. Patterns of failure after curative resection of pancreatic carcinoma. Cancer. 1990; 66: 56-61. http://dx.doi.org/10.1002/1097-0142(19900701)66:1<56::AID-CNCR2820660112>3.0.CO;2-6

[4] Van den Broeck A, Sergeant G, Ectors N, Van Steenbergen W, Aerts R, Topal B. Patterns of recurrence after curative resection of pancreatic ductal adenocarcinoma. European journal of surgical oncology: the journal of the European Society of Surgical Oncology and the British Association of Surgical Oncology. 2009; 35: 600-604. PMid: 19131205. http://dx.doi.org/10.1016/j.ejso.2008.12.006

[5] Willett CG, Czito BG, Bendell JC, Ryan DP. Locally advanced pancreatic cancer. Journal of clinical oncology: official journal of the American Society of Clinical Oncology. 2005; 23: 4538-4544. PMid: 16002845. http://dx.doi.org/10.1200/JCO.2005.23.911

[6] Shinchi H, Takao S, Noma H, et al. Length and quality of survival after external-beam radiotherapy with concurrent continuous 5-fluorouracil infusion for locally unresectable pancreatic cancer. International journal of radiation oncology, biology, physics. 2002; 53: 146-150. http://dx.doi.org/10.1016/S0360-3016(01)02806-1

[7] Koong AC, Christofferson E, Le QT, et al. Phase II study to assess the efficacy of conventionally fractionated radiotherapy followed by a stereotactic radiosurgery boost in patients with locally advanced pancreatic cancer. International journal of radiation oncology, biology, physics. 2005; 63: 320-323. PMid: 16168826. http://dx.doi.org/10.1016/j.ijrobp.2005.07.002

[8] Koong AC, Le QT, Ho A, et al. Phase I study of stereotactic radiosurgery in patients with locally advanced pancreatic cancer. International journal of radiation oncology, biology, physics. 2004; 58: 1017-1021. PMid: 15001240. http://dx.doi.org/10.1016/j.ijrobp.2003.11.004

[9] Schellenberg D, Goodman KA, Lee F, et al. Gemcitabine chemotherapy and single-fraction stereotactic body radiotherapy for locally advanced pancreatic cancer. International journal of radiation oncology, biology, physics. 2008; 72: 678-686. PMid: 18395362. http://dx.doi.org/10.1016/j.ijrobp.2008.01.051

[10] Iacobuzio-Donahue CA, Fu B, Yachida S, et al. DPC4 gene status of the primary carcinoma correlates with patterns of failure in patients with pancreatic cancer. Journal of clinical oncology: official journal of the American Society of Clinical Oncology. 2009; 27: 1806-1813. PMid: 19273710. http://dx.doi.org/10.1200/JCO.2008.17.7188 
[11] Arvold ND, Niemierko A, Mamon HJ, Fernandez-del Castillo C, Hong TS. Pancreatic cancer tumor size on CT scan versus pathologic specimen: implications for radiation treatment planning. International journal of radiation oncology, biology, physics. 2011; 80: 1383-1390. PMid: 20708856. http://dx.doi.org/10.1016/j.ijrobp.2010.04.058

[12] Furukawa H, Takayasu K, Mukai K, Inoue K, Kosuge T, Ushio K. Computed tomography of pancreatic adenocarcinoma: comparison of tumor size measured by dynamic computed tomography and histopathologic examination. Pancreas. 1996; 13: 231-235. PMid: 8884842. http://dx.doi.org/10.1097/00006676-199610000-00003

[13] Qiu H, Wild AT, Wang H, et al. Comparison of conventional and 3-dimensional computed tomography against histopathologic examination in determining pancreatic adenocarcinoma tumor size: Implications for radiation therapy planning. Radiotherapy and oncology: journal of the European Society for Therapeutic Radiology and Oncology. 2012; 104: 167-172. PMid: 22883106. http://dx.doi.org/10.1016/j.radonc.2012.07.004

[14] Hall WA, Mikell JL, Mittal P, et al. Tumor Size on Abdominal MRI Versus Pathologic Specimen in Resected Pancreatic Adenocarcinoma: Implications for Radiation Treatment Planning. International journal of radiation oncology, biology, physics. 2013 May 1; 86(1): 102-7.

[15] Nishida K, Kaneko T, Yoneda M, et al. Doubling time of serum CA 19-9 in the clinical course of patients with pancreatic cancer and its significant association with prognosis. Journal of surgical oncology. 1999; 71: 140-146. http://dx.doi.org/10.1002/(SICI)1096-9098(199907)71:3<140::AID-JSO2>3.0.CO;2-T

[16] Rezai P, Yaghmai V, Tochetto SM, et al. Change in the growth rate of localized pancreatic adenocarcinoma in response to gemcitabine, bevacizumab, and radiation therapy on MDCT. International journal of radiation oncology, biology, physics. 2011; 81: 452-459. PMid: 21570199. http://dx.doi.org/10.1016/j.ijrobp.2010.05.060

[17] Megibow AJ, Zhou XH, Rotterdam H, et al. Pancreatic adenocarcinoma: CT versus MR imaging in the evaluation of resectability--report of the Radiology Diagnostic Oncology Group. Radiology. 1995; 195: 327-332. PMid: 7724748. 